Federal Reserve Bank of Minneapolis

Research Department Staff Report 261

May 1999

\title{
Bar Codes Lead to Frequent Deliveries and Superstores*
}

\author{
Thomas J. Holmes \\ Federal Reserve Bank of Minneapolis \\ and University of Minnesota
}

\begin{abstract}
This paper explores the consequences of new information technologies, such as bar codes and computer-tracking of inventories, for the optimal organization of retail. The first result is that there is a complementarity between the new information technology and frequent deliveries. This is consistent with the recent move in the retail sector toward higher-frequency delivery schedules. The second result is that adoption of the new technology tends to increase store size. This is consistent with recent increases in store size and the success of the superstore model of retail organization.

*The views expressed herein are those of the author and not necessarily those of the Federal Reserve Bank of Minneapolis or the Federal Reserve System.
\end{abstract}




\section{Introduction}

The continuing revolution in computing technology is one of the big stories of our time. As economists, we should be interested in understanding how this revolution is affecting the organization of economic activity. Retailing is one sector for which the effects might be particularly important. Certainly the adoption of new information technology, such as bar codes and computer tracking of inventories, has been pervasive in this sector.

One impact of this new information technology is a substantial reduction in the lead time, or gap, between when inventory levels are measured and when new orders are delivered. In addition, the cost of measuring inventories has been dramatically lowered. This paper explores the effects of these factors on the optimal organization of retail. There are two main results. First, there is a complementarity between the new information technology and frequent deliveries. This is consistent with the recent move in the retail sector toward higherfrequency deliveries. Second, adoption of the new technology tends to increase store size. This is consistent with recent increases in store size and the success of the superstore model of retail organization that has been pioneered by Wal-Mart.

Consider the steps involved when a store places an order. The store takes inventory to see what is on the shelves. The inventory holdings are specified as of a point in time, for example, at the end of a particular day or at the end of a particular month. Call this point in time the measurement point. Because of lags in data collection, it may be some time after the measurement point when the store knows what its inventory was at the measurement point. The store then takes steps to place the order. This may involve time spent making decisions as well as time spent relaying information to suppliers. Then the supplier spends time processing the information to assemble the order. Finally, there is the time for the 
delivery (the delivery point). Meanwhile, during the lag between the measurement point and the delivery point, sales are taking place at the store. If a store knew what sales would be during this gap, this information would likely affect the order.

In addition to lowering the cost of inventory measurements, the new information technology has led to a dramatic reduction in the lead time between the measurement point and the delivery point. Through the use of bar codes, stores can keep track of inventories on a real time basis. Electronic data interchange (EDI) systems have been developed, connecting retailers' computers with suppliers' computers so that information passes quickly up the supply chain. ${ }^{1}$ Retailers now often make advance agreements with their suppliers on how the suppliers should react to the sales information, so the step above where retailers process information to construct the order is completely eliminated in these cases. In addition, new computer equipment allows suppliers to speed up the processing and delivery of orders. All of these effects are nicely illustrated by the relationship between Wal-Mart and Procter \& Gamble (P\&G), as discussed by Kumar (1996, p. 102):

The result [of this relationship] was a sophisticated electronic-data-interchange link, which enables $\mathrm{P} \& \mathrm{G}$ to take responsibility for managing Wal-Mart's inventory, of, say, P\&G's Pampers disposable diapers. P\&G receives continuous data by satellite on sales, inventory, and prices for different sizes of Pampers at individual Wal-Mart stores. This information allows $\mathrm{P} \& \mathrm{G}$ to anticipate Pampers sales at Wal-Mart, determine the . . quantity required, and automatically ship the orders.

\footnotetext{
${ }^{1}$ References on these various technologies include Kinsey et al. (1996); Brown (1997); Dunlop and Rivkin (1997); Kahn and McAlister (1997); and textbooks on logistics, including Coyle, Bardi, and Langley (1996).
} 


\section{Discussion of Results}

To understand the first result of a complementarity between the new information technology and higher-frequency delivery, consider the following example of stores that sell toothpaste. Suppose that in the previous technological environment, when an order was placed at the end of the day, the toothpaste sales that occurred during the day were unknown. Now, with the advent of bar codes and related technology, the sales that have occurred during the day are known at the end of the day when the order is placed. For the first result, delivery frequency is taken as exogenous. Compare two stores: a store that gets monthly deliveries of toothpaste and a store that gets daily deliveries. Which store values the new technology more?

There are two benefits associated with the new information technology. The first is that the cost of measuring inventory falls. The daily-delivery store obviously values this benefit more than the monthly-delivery store. In fact, the daily-delivery store values this benefit 30 times more because the daily-delivery store takes 30 measurements a month compared to one a month for the monthly-delivery store. The second benefit is that there is more information at the time an order is placed, so the store can make a better order decision. Simple intuition might suggest that the daily-delivery store values the benefit of improved decision making more as well, perhaps even 30 times more, since the daily-delivery store makes 30 decisions a month compared with the monthly-delivery store's one. However, while this simple intuition captures something, it does not get the whole story.

On one hand, this simple comparison can overstate the daily-delivery store's benefit of improved decision making compared with the monthly-delivery store. In fact, in one special case of the model, the benefit is exactly the same for both store types. In this special case, 
every potential sale is so important that the probability of a stockout is set to zero. In the old days without bar codes, when the store with monthly deliveries placed its order, it did not know whether a tube of toothpaste was sold during the day of the order, so it had to order an extra tube, just in case. If it turned out that no such tube had been sold on order day, the store would be carrying an extra tube in its inventory throughout its one-month order cycle. Now, with the advent of bar codes, the store can avoid paying a month's worth of inventory carrying costs for this tube of toothpaste. Even though the monthly-delivery store utilizes bar codes once for every 30 times that the daily-delivery store uses them, the value of the improved decision making for each use is 30 times as great.

On the other hand, the above simple comparison can also understate the relative benefit to the daily-delivery store. This can occur when the parameters are such that there is some probability of stockouts. When the monthly-delivery store puts in an order, it trades off the marginal inventory carrying cost of one extra tube with the marginal benefit of a reduction in the stockout probability over the upcoming month. With the advent of bar codes, if a store sells no toothpaste on order day, it might utilize this information and order one less tube. However, the value of this information may be relatively small since the reduction in inventory cost from one less tube is offset by the higher stockout probability from one less tube. In contrast, the daily-delivery store will be running a tight ship, closely matching orders to sales. The information about any one day's sales may be more valuable to the daily-delivery store than to the monthly-delivery store. But on top of this, the daily-delivery store uses the information 30 times a month compared with the other store's one-time use.

The second result is that the advent of the new technology increases store size. For the second result, both store size and delivery frequency are taken as endogenous. The advent 
of bar codes increases the optimal delivery frequency. Given economies of scale in delivery, the optimal store size also increases. In simple terms, it may not be economical to have half-empty trucks making daily deliveries. So stores get bigger to fill up the trucks.

There is great interest in understanding the success of superstores, such as Wal-Mart, and a variety of explanations have been given. ${ }^{2}$ The second result identifies a new factor that has contributed to the success of this format. In the days before bar codes, a small town might have had five stores, each specializing in a particular product line: that is, a hardware store, a clothing store, a pharmacy, and so forth. Each of these stores might have received a delivery once a week: that is, on Monday a truck would have come in with hardware, on Tuesday a truck would have come in with clothing, and so forth. In this earlier period, there might have existed some cost savings in combining these product lines into the same store, but these advantages would have been offset by disadvantages, such as the store losing focus or the parking lot becoming too big. Now, with the advent of bar codes, it has become beneficial to increase delivery frequency. This has been accomplished by replacing the five specialized stores with a Wal-Mart that carries all five product lines. There may be one truck that arrives in town each day on Monday through Friday, but now this truck comes from Wal-Mart's central warehouse and has on each day the hardware, the clothing, and so forth that are needed to replenish the shelves after each day's sales.

\section{Related Literature}

The order decision model considered here is a textbook problem in inventory man-

\footnotetext{
${ }^{2}$ Bagwell, Ramey, and Spulber (1997) emphasize the presence of increasing returns in the adoption of new technology. An alternative idea is that the adoption of the new information technology may require greater coordination of various stages of distribution; such coordination may be easier within the same firm and can potentially account for Wal-Mart's vertical integration into wholesaling activities.
} 
agement. In the operations research literature, there is a great interest in how reductions in order lead time affect optimal inventory holdings. (See Song 1994, for example.) However, this literature does not analyze how a reduction in lead time affects the optimal delivery frequency and the optimal store size.

The complementarity identified in the first result is very much in the spirit of Milgrom and Roberts (1990). ${ }^{3}$ They discuss a number of new practices that are being adopted in modern manufacturing, such as computer aided design, smaller batch sizes, shorter productdevelopment times, quicker order processing, and speedier delivery. They show that these various activities are complementary. My model is substantially different from their model. For example, my model is of a retail establishment that permanently loses sales when stockouts occur. Their model is of a manufacturer that takes orders. They have a variable called order-processing time and another variable called delivery time that apply to orders their manufacturing establishment receives. They show that there are gains to reducing both. The manufacturer's demand depends upon total time between initial order and final delivery and is a decreasing convex function of total time. Hence, a reduction in order-processing time increases the marginal benefit of a shorter delivery lag. This source of complementarity is fundamentally different from the source of complementarity between shorter lead time and more frequent deliveries identified in my paper.

Two recent empirical studies, Abernathy et al. (1995) and Hwang and Weil (1998), examine the hypothesis of a complementarity between short lead times and frequent delivery (Result 1 of this paper). Abernathy et al. (1995) stress the importance of these changes in the retail sector and use the term lean-retailing to refer to the adoption of these new information

\footnotetext{
${ }^{3}$ See also Milgrom and Roberts (1988), which shows how information and inventories are substitutes.
} 
practices in conjunction with higher-frequency deliveries. Both of the above mentioned studies examine data on apparel suppliers. Suppliers are classified as either high- or low-frequency suppliers. The studies find that suppliers making frequent deliveries are substantially more likely to make use of the new information technologies in their relationships with retailers. For example, Hwang and Weil (1998) report that in 1988, 70 percent of high-frequency suppliers were putting bar codes on their products while only 38 percent of low-frequency suppliers were doing so. There were analogous differences in the use of EDI. In addition to this crosssection evidence, there is strong evidence of this relationship in the time series. There was a substantial increase in the use of the new information technologies over the period from 1988 to 1992. In fact, Hwang and Weil (1998) report that use of EDI increased from 33 percent to 83 percent over this four-year period. Over this same period, there was a substantial increase in delivery frequency. Whereas in 1988 only 7 percent of sales where delivered on a weekly frequency, by 1992 the fraction of such sales had increased to 30 percent.

At this point, there is little formal evidence on the second result of the link between the new information technologies and store size. However, the anecdotal evidence concerning Wal-Mart and other superstores, such as Home Depot, is consistent with this link. The emergence of these large stores has increased store sizes, particularly in rural areas. ${ }^{4}$ It is well known that superstore chains, such as Wal-Mart and Home Depot, have been pioneers of the new information technologies. They have also been leaders in increasing the frequency of deliveries. For example, according to Vance and Scott (1994, p. 93), "Its insistence on the

\footnotetext{
${ }^{4} \mathrm{My}$ analysis of County Business Patterns data (U.S. Bureau of the Census 1981, 1997) indicates that the percentage of retail employment in establishments with 20 or more employees increased from 57 percent in 1977 to 67 percent in 1995. In the building materials sector that is now dominated by Home Depot, the share increased from 35 to 57 percent over the same period.
} 
frequent, often daily delivery of merchandise set Wal-Mart apart from rivals such as K-Mart, which generally delivered merchandise to its stores once every five days." In the model, stores get bigger to exploit increasing returns on deliveries. This effect has quantitative significance only to the extent that the resources allocated to deliveries are significant. In 1992, the resource cost of the transportation of goods for the trade sector (wholesale and retail) was $\$ 52$ billion, and this accounted for 4.7 percent of the value of output of the sector. ${ }^{5}$ This significant sum suggests that the movement from weekly and monthly deliveries to daily deliveries that is taking place in the retail sector might entail substantial costs if the structure of retail were left alone. Increasing store size may be an important way of economizing on these costs.

\section{Organization}

The environment considered in this paper is one where there is stochastic demand, a given order lead time, and orders that are delivered at regular intervals, such as once a week or once a month. Section 2 develops the model of the order decision for a particular product, holding the lead time for the order and the delivery frequency fixed. The advent of bar codes and related information technology in this environment is modeled as a simultaneous reduction in lead time and inventory measurement costs. Section 3 shows that the value of bar codes increases with the exogenous delivery frequency. Section 4 imbeds the order-decision model into a model of a store where order frequency and store size are both endogenous. Section 5 concludes.

\footnotetext{
${ }^{5}$ This figure includes the imputed cost of in-house trucking services as well as hired trucking services. The value of retail output is sales less the cost of goods sold. The figure here is from the U.S. Transportation Satellite Accounts for 1992 that have recently been constructed (Fang et al. 1998). This is the first attempt to account for the value of in-house transportation services, for example, deliveries by Wal-Mart employees. In-house services account for more than 80 percent of the total.
} 


\section{The Order-Decision Model}

This section presents a model of an order decision for a particular product where deliveries are at fixed intervals.

Time is discrete, $t=\{0,1,2, \ldots\}$. In period $t$, there is a realization of demand $z_{t}$ for the product that is drawn i.i.d. over time from the unit interval, $z_{t} \in[0,1]$. The distribution of this random demand is $F(\cdot)$, and the continuous density is $f\left(z_{t}\right)$.

Let $h$ denote the fixed interval length of the order cycle. For simplicity, two cases will be considered, $h=1$ and $h=2$. With $h=1$, there is a delivery each period. With $h=2$, there is a delivery every other period. The order cycle length $h$ is exogenous here, although later it will be a choice variable.

Assume that the consumer reservation price is $\alpha$ and that the price of the good is fixed at this level. Assume that the wholesale cost is $\omega$ per unit. There is a physical cost $\lambda$ of holding a unit of inventory in a period. Assume that $\alpha>\omega+\lambda$ so that the retail price exceeds the sum of the wholesale cost and the physical holding cost. The discount factor is $\beta<1$.

In each period $t$, the store begins the period with an inventory stock $s_{t}$. If there is a delivery scheduled for the period, the truck arrives with $x_{t}$ units of product, and this increases the inventory on hand to $r_{t}=s_{t}+x_{t}$. The sales for period $t$ are drawn out of the stock $r_{t}$ that includes the current period's delivery. Given the demand realization $z_{t}$, the quantity of sales $q_{t}$ in the period is the minimum of demand and the amount of goods in stock,

$$
q_{t}=\min \left\{r_{t}, z_{t}\right\} .
$$

The store begins the next period with whatever is left over after these sales, $s_{t+1}=r_{t}-q_{t}$. 
The store places an order in period $t$ for delivery in period $t$. There is lag of $\ell$ in the information the store has when the order is placed. At this point, the store has knowledge of demand in period $t-\ell$. For example, if $\ell=1$, the store knows the sales realization $z_{t-1}$ in period $t-1$ when placing the order for delivery in period $t$. If $\ell=0$, the store knows the sales realization $z_{t}$ in period $t$ when placing the order for delivery in period $t$. The lag $\ell$ will be referred to as the lead time to be consistent with the literature.

There is a cost $\gamma$ of measuring inventory that must be incurred every time an order is made.

Technological change is modeled as a simultaneous reduction in the lead time $\ell$ and the inventory measurement cost $\gamma$. There are two regimes, the no-bar-code case and the barcode case. Let $j \in\{N, B\}$ indicate two regimes. The assumption is that $\ell^{B}<\ell^{N}$ and that $\gamma^{B}<\gamma^{N}$. While the new technology is referred to as bar codes, it is meant to be interpreted more broadly to include the wide range of the new information technologies being adopted by the retail sector, including electronic data interchange.

To make the analysis analytically tractable, I focus on the simplest case, where $\ell^{B}=0$ and $\ell^{N}=1$. The case where the lead time $\ell \geq 2$ is relatively complicated. I have looked at the case where $\ell=2$ in numerical examples. In these numerical examples, the effects of reducing the information lag from $\ell=2$ to $\ell=1$ are qualitatively the same as the effects of reducing the lag from $\ell=1$ to $\ell=0$.

The initial period is $t=0$. The store begins this initial period with empty shelves, $s_{0}=0$, and this is known when the order is placed for delivery in the initial period. 


\section{A. The No-Bar-Code Case}

This subsection analyzes the no-bar-code case, where the lead time is $\ell^{N}=1$. Recall that there are two possible order cycle lengths, $h=1$ and $h=2$. Optimal behavior is determined for each case.

Suppose first that $h=1$ so that there is a delivery in every period. Each period begins with $s_{t}$ units of the good on the shelves. The store places an order for $x_{t}$ units of the good for delivery in the current period before the customers arrive. Since there are no bar codes, the store places this order before the realization of $z_{t}$. However, the store does observe $z_{t-1}$ and so can observe $s_{t}$. Let $\tilde{v}_{1}^{N}(s)$ be the discounted value of optimal behavior given the state s. The superscript $N$ denotes that this is the no-bar-code case. The subscript denotes that the cycle length is $h=1$. This function solves

$$
\begin{aligned}
\tilde{v}_{1}^{N}(s)= & \max _{x}-\omega x-\lambda(s+x)-\gamma^{N}+\int_{0}^{s+x} f(z)\left(\alpha z+\beta \tilde{v}_{1}^{N}(s+x-z)\right) d z \\
& +(1-F(s+x))\left(\alpha(s+x)+\beta \tilde{v}_{1}^{N}(0)\right) .
\end{aligned}
$$

The store picks an order size $x$. The first term accounts for the wholesale cost of the order $x$. The second term is the physical cost of holding $s+x$ in inventory for the period. The third term subtracts the cost $\gamma^{N}$ of measuring inventory. The fourth term accounts for the possibility that realized demand $z$ is less than the stock on the shelves $s+x$ so that sales in the period are $z$ and the next period begins with $s+x-z$ on the shelves. The final term accounts for the return in the event of a stockout.

Analysis of this problem is simplified by noting that along the optimal path, it will in every period be optimal to add to the inventory (set a positive $x$ ). If I assume that the store 
returns the unsold merchandise $s_{t}$ at the beginning of the period for the wholesale price $\omega$ and then chooses how much inventory to buy (starting from inventory equal to zero), I get the same solution as in a problem where returns are infeasible. The advantage of looking at this problem in this other way is that it reduces the analysis to a static problem. In this static problem, the store picks an order size $r$ (starting from zero) that satisfies the following problem:

$$
y_{1}^{N}=\max _{r}-(\omega+\lambda) r-\gamma^{N}+\int_{0}^{r} f(z)(\alpha z+\beta \omega(r-z)) d z+(1-F(r)) \alpha r .
$$

This problem is simpler than (1) since there is no value function $\tilde{v}$ buried in it. If sales $z$ are less than the inventories $r$, the excess $r-z$ is returned in the next period at a present value of $\beta \omega$ per unit. The present discounted value of these payoffs in the initial period where $s_{0}=0$ is

$$
v_{1}^{N}=\frac{1}{1-\beta} y_{1}^{N}
$$

Next consider the case where $h=2$ so that deliveries are at alternating periods. Analogous to the case where $h=1$, it is possible to look at a particular order cycle and determine the optimal order, taking as given that unsold items are returned at the end of the cycle:

$$
y_{2}^{N}=\max _{r}-(\omega+\lambda) r-\gamma^{N}+\int_{0}^{r} f(z)(\alpha z+\beta \hat{v}(r-z)) d z+(1-F(r)) \alpha r
$$

where $\hat{v}(s)$ is defined to be the payoff of going into the second period of the order cycle with $s$ units:

$$
\hat{v}(s)=-\lambda s+\int_{0}^{s} f(z)(\alpha z+\beta \omega(s-z)) d z+(1-F(s)) \alpha s .
$$


Note that the optimal order $r$ solving (4) may exceed one (the maximum value of $z$ ), in which case, the integral in (4) is evaluated for $z \in[0,1]$ (since $f(z)=0$ for $z>1$ ) and the third term of (4) (the stockout term) is zero (since $F(r)=1$ for $r \geq 1$ ).

The discounted value to the store is the discounted value of these two-period order cycles,

$$
v_{2}^{N}=\frac{1}{1-\beta^{2}} y_{2}^{N} .
$$

Note that $\beta$ is squared because the cycle length is $h=2$.

\section{B. The Bar-Code Case}

Now consider the bar-code case where $\ell=0$. If $h=1$ so that there are deliveries every period, the optimal policy is obvious. The store has advance information as to the realization $z_{t}$ of sales for the period, so the order should be set so that the stock on hand $r_{t}$ exactly equals sales. No excess inventories are held. The maximum expected return with bar codes over the one-period order cycle is then

$$
y_{1}^{B}=\int_{0}^{1} f(z) z(\alpha-\omega-\lambda) d z-\gamma^{B} .
$$

Now consider the alternating delivery case, $h=2$. The maximum expected two-period return over the order cycle is

$$
\begin{aligned}
y_{2}^{B}= & \max _{s_{1}} \int_{0}^{1} f(z)\left[-(\omega+\lambda)\left(z+s_{1}\right)-\gamma^{B}+\alpha z\right] d z \\
& +\beta\left(-\lambda s_{1}+\int_{0}^{s_{1}} f(\tilde{z})\left(\alpha \tilde{z}+\beta \omega\left(s_{1}-\tilde{z}\right)\right) d \tilde{z}+\left(1-F\left(s_{1}\right)\right) \alpha s_{1}\right) .
\end{aligned}
$$

The realization of demand in the current period is $z$ and in the following period is $\tilde{z}$. The firm knows the current realization $z$ when it places the order and orders to meet this demand. 
It also orders an additional amount $s_{1}$ to take as inventory into the following period, so the total order size is $x=z+s_{1}$.

\section{The Value of Bar Codes}

The benefit of bar codes over the first two periods for the $h=2$ case is

$$
\Delta_{2} \equiv y_{2}^{B}-y_{2}^{N} .
$$

The benefit of bar codes for $h=1$ over the first two periods is

$$
\Delta_{1} \equiv(1+\beta)\left[y_{1}^{B}-y_{1}^{N}\right],
$$

the benefit of using bar codes in the initial period plus the benefit in the subsequent period. This section shows that $\Delta_{1}>\Delta_{2}$; that is, that over the first two periods, the $h=1$ case values bar codes more than the $h=2$ case. Since the return in future periods is just a repeat of the return in the first two periods, this is equivalent to showing that the comparison holds for the infinite horizon return.

The advent of bar codes has two effects. It reduces the measurement $\operatorname{cost}\left(\gamma^{B}<\gamma^{N}\right)$, and it reduces the lead time $\left(\ell^{B}<\ell^{N}\right)$. The benefit of the savings in measurement costs is obviously greater in the $h=1$ case because over the two-period interval that we are focusing on, two measurements are taken in the $h=1$ case while only one measurement is taken in the $h=2$ case. The analysis of the benefit from the reduction in lead time is more subtle. To focus attention on this latter effect, it is assumed for the rest of this section that bar codes have no effect on measurement cost, $\gamma^{B}=\gamma^{N}=0$.

It is difficult to make these comparisons for general versions of the model. To make progress, two special cases are considered. In the first case, there are two discrete possible realizations of demand in each period. In the second case the demand realization is uniform. 


\section{A. Discrete Demand}

This subsection considers the analytically tractable case where demand is discrete and takes on two extreme values. Suppose that $z_{t}=1$ with probability $f_{1}$ and that $z_{t}=0$ with probability $f_{0}=1-f_{1}$. This subsection determines how the solution varies over the range of $\alpha$ and compares the value of bar codes for the $h=1$ and $h=2$ cases. There are three main results. First, over the entire parameter space, the value of bar codes to the $h=1$ case is greater or equal to the value to the $h=2$ case. Second, for high enough $\alpha$, where the probability of stockouts is zero, the relationship holds with equality. Third, when there is some probability of a stockout, the value to the $h=1$ case can be substantially greater than to the $h=2$ case.

There are four critical levels of $\alpha$ in the solution:

$$
\begin{aligned}
\alpha^{\circ} & \equiv \omega+\lambda \\
\alpha^{\prime} & \equiv \frac{\omega+\lambda-f_{0} \beta \omega}{f_{1}} \\
\alpha^{\prime \prime} & \equiv \frac{\omega+(1+\beta) \lambda-f_{0} \beta^{2} \omega}{\beta f_{1}} \\
\alpha^{\prime \prime \prime} & \equiv \frac{\omega+(1+\beta) \lambda-\left(1-f_{1}^{2}\right) \beta^{2} \omega}{\beta f_{1}^{2}} .
\end{aligned}
$$

Consider first the case where $h=1$. Suppose the store has bar codes. The rule here is to order a good if $z_{t}=1$, as long as $\alpha$ is greater than the critical point $\alpha^{\circ}$. This critical point $\alpha^{\circ}$ is defined so that profit in the event of a sale is exactly zero. Without bar codes, if the store places an order and there is no sale $\left(z_{t}=0\right)$, the store loses the physical and interest cost of holding the inventory. The point $\alpha^{\prime}$ is defined so that the expected profits in the event 


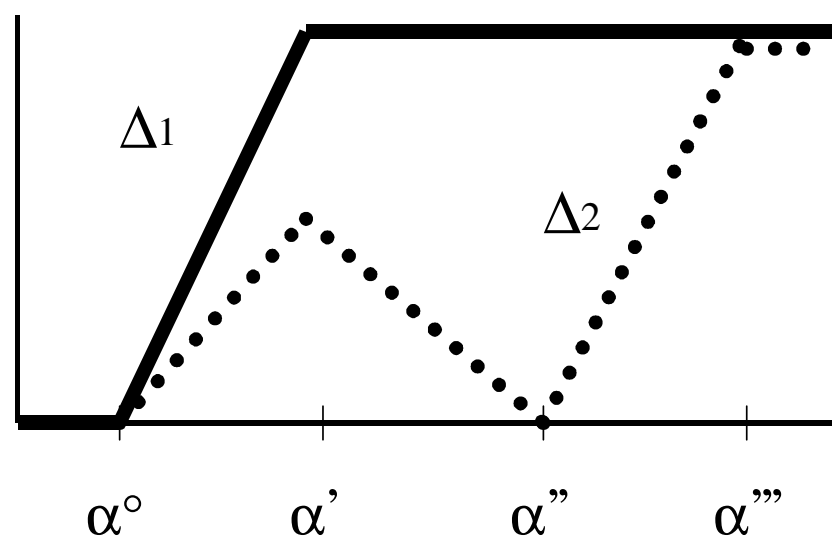

Figure 1: The Value of Bar Codes for $h=1$ and $h=2$

of a sale exactly offset the expected losses in the event of no sale,

$$
f_{1}\left(\alpha^{\prime}-\omega-\lambda\right) \equiv f_{0}(\omega+\lambda-\beta \omega)
$$

If $\alpha<\alpha^{\prime}$, then without bar codes, the store never orders. If $\alpha>\alpha^{\prime}$, the store makes sure there is always one unit of the good in stock.

The solid line in Figure 1 illustrates the value $\Delta_{1}$ of bar codes to $h=1$ over a twoperiod horizon. For $\alpha<\alpha^{\circ}$, no orders are placed with or without bar codes, so $\Delta_{1}=0$. For $\alpha \in\left(\alpha^{\circ}, \alpha^{\prime}\right)$, the payoff is zero without bar codes, so the change in payoff equals the bar code expected payoff (over two periods),

$$
\Delta_{1}=(1+\beta) f_{1}(\alpha-\omega-\lambda)
$$

For $\alpha>\alpha^{\prime}$, there is always a sale with or without bar codes. The benefit of bar codes is then

$$
\Delta_{1}=(1+\beta) f_{0}(\lambda+(1-\beta) \omega) .
$$

This is the elimination (over two periods) of the expected loss from the physical and interest cost of holding inventory in the event that no consumer shows up. 
Now consider the case where $h=2$. If there are no bar codes and $\alpha=\alpha^{\prime}$, the store is indifferent between shutting down and ordering one unit, just as in the $h=1$ case. For $\alpha \in\left(\alpha^{\circ}, \alpha^{\prime}\right)$, the optimal policy with bar codes is to order one unit at the order point if there is a certain sale $\left(z_{t}=1\right)$. The value of bar codes in this region of $\alpha$ (over the two-period horizon) is

$$
\Delta_{2}=f_{1}(\alpha-\omega-\lambda) .
$$

This is illustrated in Figure 1 by the dotted line. The value $\Delta_{1}$ for the case where $h=1$ exceeds $\Delta_{2}$ in this range by a factor of $(1+\beta)$ because in the $h=1$ case, the benefit is obtained every period, while in the $h=2$ case, the benefit is obtained only at alternating order periods.

As mentioned, if $\alpha=\alpha^{\prime}$ and there are bar codes, the optimal policy is to order a single unit whenever there is a certain sale in the order period. Thus, there are no sales in the subsequent period. If $\alpha$ is increased, eventually the point $\alpha^{\prime \prime}$ is reached, defined to be where the store is indifferent to ordering so as to have a unit in stock in the subsequent period.

A key point is that at $\alpha=\alpha^{\prime \prime}$, the value of bar codes for $h=2$ is zero; that is, $\Delta_{2}=0$. The optimal policy with no bar codes is to order so that there is one good in stock at the beginning of the cycle. If it turns out that the consumer shows up in the initial period, then the store is out of stock at the beginning of the second period. But that is not a problem since with bar codes, the store is indifferent to ordering in that case.

The last critical point is $\alpha^{\prime \prime \prime}$. At this point, if $h=2$ and there are no bar codes, the store is just indifferent between ordering to have one unit in stock and ordering to have two units in stock. For $\alpha>\alpha^{\prime \prime \prime}$, the store orders to have two units, and the probability of a 
stockout is zero. In the event that there is no sale in the first period, the store could have ordered to have one unit in stock and still avoided a stockout. With bar codes, the store could have avoided ordering this extra unit of inventory and paying the cost of carrying it for two periods. The benefit of bar codes is then

$$
\begin{aligned}
\Delta_{2} & =f_{0}\left(\lambda+\beta \lambda+\left(1-\beta^{2}\right) \omega\right), \text { for } \alpha>\alpha^{\prime \prime} \\
& =f_{0}(1+\beta)(\lambda+(1-\beta) \omega) .
\end{aligned}
$$

This top line shows the benefit as the probability $f_{0}$ that there is no sale in period one times the sum of the physical cost $(1+\beta) \lambda$ of holding the extra unit two periods plus the interest cost. The second line manipulates the equation to reveal that the benefit in this case is exactly the benefit from (8) for $h=1$ for $\alpha$ in this range. The $h=2$ case uses bar codes only half as often as $h=1$, but each use of the bar codes is worth $1+\beta$ times as much, so the benefit is the same.

The discussion above shows how the benefit of bar codes is calculated at the critical points. Between the critical points, the benefit is linear in $\alpha$, as illustrated in Figure 1. The comparison between $\Delta_{1}$ and $\Delta_{2}$ can be summarized as follows:

Result 1 (Discrete Case). For the entire range of the parameter space, $\Delta_{1} \geq \Delta_{2}$, so bar codes and frequent delivery are (weakly) complementary. For $\alpha>\alpha^{\prime \prime \prime}$, then, $\Delta_{1}=\Delta_{2}$. In this range, $\alpha$ is so high, there is never any probability of a stockout. If $\alpha<\alpha^{\prime \prime \prime}$, then there is strict complementarity, $\Delta_{1}>\Delta_{2}$. For $\alpha$ close to $\alpha^{\prime \prime}, \Delta_{2}$ is close to zero. 


\section{B. The Uniform Distribution}

Now return to the case where the sales realization is continuous, but restrict it to be a uniform distribution to make the analysis tractable, that is, $F(z)=z$. The result of this section is that the value of bar codes for the frequent-delivery case exceeds that for the infrequent-delivery case. In a large portion of the parameter space, the difference exceeds a factor of $1+\beta$. Not only does the frequent-delivery case utilize bar codes on more occasions, but in this region, the average increment in value of the improved decision making is greater for each use of bar codes.

Normalize the sale price to $\alpha=1$. This leaves three parameters: the wholesale price $\omega$, the holding cost $\lambda$ (note that the sum $\omega+\lambda$ is less than the retail price of one), and $\beta$. Numerical analysis yields the following result.

Result 1 (Uniform Case). The value of bar codes is strictly greater in the $h=1$ case than in the $h=2$ case; that is, $\Delta_{1}>\Delta_{2}$. For each $\beta$ and $\lambda$ there exists a critical level wholesale cost $\tilde{\omega}(\beta, \lambda)$ such that if $\omega>\tilde{\omega}(\beta, \lambda)$ then $\Delta_{1}>(1+\beta) \Delta_{2}$.

The critical point $\tilde{\omega}$ is illustrated in Figure 2 as a function of $\beta$ of several values of $\lambda$. The region above $\tilde{\omega}$ is where the value to $h=1$ exceeds a factor of $1+\beta$. For $\lambda=0$, this region contains the bulk of the $(\beta, \omega)$ space. For higher $\lambda$, the region is even bigger. For example, for $\lambda=0.04$ (admittedly a large value), the region contains all but a small piece on the bottom right-hand side.

The results of this subsection are consistent with the results from the previous subsection. There, the difference exceeded a factor of $(1+\beta)$ when $\alpha$ was low and the probability of a stockout was high. Here, this is true when $\omega$ is high, when $\lambda$ is large, and when $\beta$ is small. The probability of a stockout is relatively high in all three cases. In the previous example, 


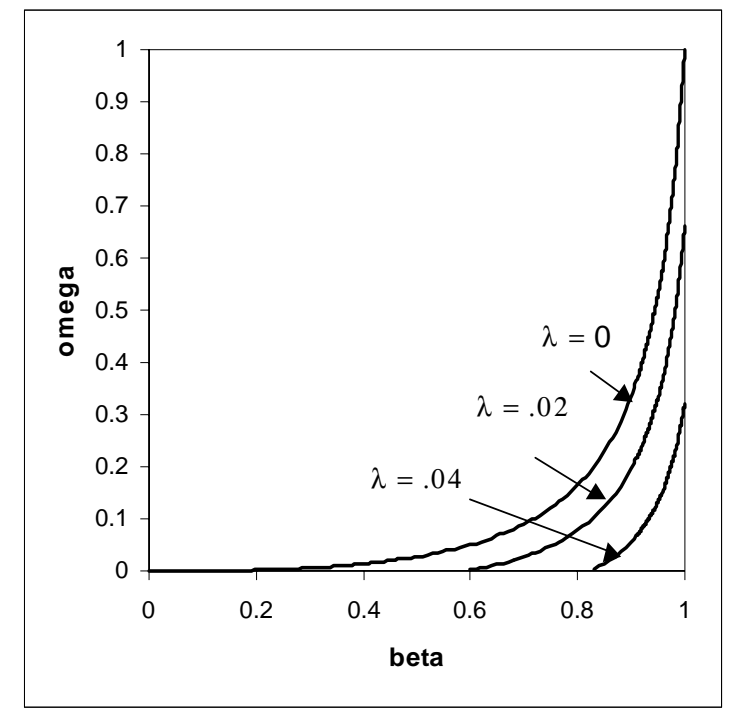

Figure 2: Critical Levels of $\omega$ (for various $\lambda$ )

there was some range of the parameter space where the value of bar codes was exactly the same for the two order cycles. This occurred in the region where the probability of a stockout was zero. Here, there is always some probability of a stockout, and the inequality is strict.

\section{Superstores and Delivery Frequency}

This section imbeds the model of an order decision for a particular product analyzed above into a model of a store where the choice of products carried and the delivery frequency are both endogenous variables. As before, the advent of bar codes leads to a reduction in the order lead time, $\ell^{N}$ to $\ell^{B}$, and to a reduction in the inventory measurement cost, $\gamma^{N}$ to $\gamma^{B}$. The question addressed is how the new technology affects the organization of the store.

\section{A. The Model of a Store}

There is a continuum of separate products. Products are indexed by $i$ on the interval $i \in[0, \mu]$ so that $\mu$ is the measure of the set of products. 
A store offers some subset of the set of products. Let $m \leq \mu$ denote the measure or number of products that a store sells. A store also selects a delivery frequency $h$. All the different goods come in the same truck, so the delivery frequency is the same for all goods.

A key aspect of the model here is that there are economies of scale in delivery. For simplicity, I make the extreme assumption that there is a fixed cost of $\phi$ for a delivery and that this cost does not vary with the size of the delivery or with the number of products $m$ that are delivered. Let $c_{D}(h)$ be the present value of delivery costs at order frequency $h$. As before, there will be two order cycle lengths: daily delivery $h=1$ and alternating delivery $h=2$. Discounted delivery costs for these two cases are

$$
\begin{aligned}
c_{D}(1) & =\frac{1}{1-\beta} \phi \\
c_{D}(2) & =\frac{1}{1-\beta^{2}} \phi .
\end{aligned}
$$

Given the economies of scale in delivery, there will be an incentive to make stores big. To offset this, I assume that there is some other source of diseconomies of scale. Let $c_{S}(m)$ be this other cost, which I will call the setup cost. Suppose that this is a cost that must be paid for each product. Assume that the $\operatorname{cost} c_{S}(m)$ is increasing and convex. This increasing cost captures factors that limit the size of a store. These factors include unwieldy parking lots, increasing transportation cost from drawing from a wider market area, management diseconomies, and so forth.

As in the earlier analysis, equation $(3), v^{j}(h)$ is the (maximized) discounted operating product on each product. It equals discounted revenues on the product less the costs of the product described in Section 2. These costs include the wholesale cost, the physical and 
interest costs of inventory, and the cost of measuring inventory. If there is a proportionate change in the number of products, everything else fixed, there is a proportionate change in these revenues and costs. In particular, if there are twice as many different products, the cost of measuring inventory is assumed to double.

The total discounted profit of a store selling $m$ different products with an order-length interval of $h$, given the level of technology $j$, is

$$
\pi^{j}(m, h)=m v^{j}(h)-c_{D}(h)-m c_{S}(m), j \in\{B, N\} .
$$

The first term is the store's number of products $m$ times the product-level profit. The second term subtracts the discounted value of the cost of the sequence of deliveries. This is not multiplied by $m$ because of the scale economies. The third term subtracts the setup costs that must be paid for each of the $m$ products

Two formulations of the objective function are considered. The first formulation is a social planner's problem. Suppose that the $\alpha$ parameter represents the gross benefit to society when a consumer arrives at a store and obtains a unit of product that he or she desires. Then the $\pi$ function as defined above represents the net social surplus created by a store of size $m$ and order length $h$. Suppose that the task of the planner is to take the total set of products $\mu$ and divide this set into different stores of size $m$. A choice of store size $m$ implies that the number of different stores is $n=\frac{\mu}{m}$. (Allow the number of stores to be a continuous variable.) The objective of the planner is to maximize the total surplus. This is equivalent to maximizing total surplus per product (since that number of products is fixed at $\mu$ ). Surplus per product is obtained by dividing $\pi$ above by $m$ :

$$
w^{j}(m, h)=v^{j}(h)-\frac{c_{D}(h)}{m}-c_{S}(m), j \in\{B, N\} .
$$


The problem of the planner is to pick a store size $m \leq \mu$ and an order length $h \in\{1,2\}$ to maximize the above, given the level of technology $j$,

The second formulation of the objective function is that of a store maximizing the profit (11).

\section{B. The Effect of Bar Codes}

The result of this section is the following:

Result 2. With the advent of bar codes, the optimal order length $h$ (weakly) decreases. The optimal number of products in the store increases if the order length decreases (and stays the same if the order length stays the same).

The proof is a direct consequence of the earlier result about the complementarity of bar codes and delivery frequency. Suppose that initially there are no bar codes and that the optimal order interval is $h=2$. Earlier it was shown that bar codes are more valuable with a shorter delivery cycle,

$$
v^{B}(1)-v^{N}(1)>v^{B}(2)-v^{N}(2)
$$

or, equivalently,

$$
v^{B}(1)-v^{B}(2)>v^{N}(1)-v^{N}(2) .
$$

Since the benefits of reducing the delivery cycle to $h=1$ are greater but cost remains the same, it may now be optimal to reduce the delivery cycle to $h=1$.

But consider how the choice of $h$ affects the choice of store size $m$. For the social planner, the first-order condition for the choice of store size is

$$
\frac{\partial w^{j}(m, h)}{\partial m}=\frac{c_{D}(h)}{m^{2}}-c_{S}^{\prime}(m)=0
$$


The choice of $m$ balances the increasing returns to scale for deliveries (the first term) against the decreasing returns from other factors (the second term). A reduction in the cycle length $h$ increases the first term, and the optimal store size $m$ increases.

\section{An Alternative Interpretation of Store Size}

In the discussion so far, $m$ has been defined as the number of products that a store carries. The variable $m$ can also be interpreted as a scale parameter in a model with a single product; that is, $m$ is simply the number of customers that a store serves. But interpreting it in this way requires some additional discussion.

Recall that demand in the period is a random draw $z_{t}$ from some distribution $F\left(z_{t}\right)$ on the unit interval. If an increase in scale $m$ is a multiplicative parameter times the original demand $z_{t}$, then the argument goes through. That is, it works when demand is $m z_{t}$, where $z_{t}$ is drawn from $F(z)$. Here, increases in scale do not allow for any law of large numbers effect. The uncertainty is at the aggregate level, so increasing scale does not get rid of it.

Suppose instead that when the store scales up it pools the random demand from $m$ individual consumers; that is, demand in period $t$ is $\sum_{i=1}^{m} z_{i t}$, where for each customer $i$ the demand $z_{i t}$ is drawn i.i.d. from $F\left(z_{i t}\right)$. In this case, when the store is large, the idiosyncratic demands of individual customers will average out and demand will be predictable through the law of large numbers. The benefit of bar codes will be negligible for such a store compared with the benefit (per unit of sales) for small stores. In such a case, the advent of bar codes can tilt the balance toward small stores. 


\section{Conclusion}

This paper shows that frequent deliveries complement the adoption of new information technologies. Given economies of scale in delivery, increasing store size is one way to accommodate increases in deliveries. Of course, there are other ways to achieve more frequent deliveries besides making stores bigger. For example, increased coordination of deliveries to establishments that are in the same geographic area may facilitate increases in delivery frequency. Chains of convenience stores that blanket an area may serve the function of increasing the coordination of deliveries. As another example, expansion of package delivery services like Federal Express may permit even small stores to work on a just-in-time delivery system.

Pressures to increase store size may differ between urban and rural areas. In urban areas, a high concentration of establishments in the same area may make it possible to obtain economies of scale in frequent deliveries without making establishments bigger. In rural areas, these economies of scale may only be available through expansion of store size. In this context, it is interesting to note that Wal-Mart's emergence in rural areas preceded its emergence in urban areas. 


\section{References}

Abernathy, Frederick H.; John T. Dunlop; Janice H. Hammond; and David Weil (1995), "The Information-Integrated Channel: A Study of the U.S. Apparel Industry in Transition," Brookings Papers on Economic Activity (Microeconomics 1), 175-246.

Bagwell, Kyle; Garey Ramey; and Daniel F. Spulber (1997), "Dynamic Retail Price and Investment Competition," RAND Journal of Economics 28(2), 207-27.

Brown, Stephen A. (1997), Revolution at the Checkout Counter, Harvard University Wertheim Committee: Cambridge, Mass.

Coyle, John J.; Edward J. Bardi; and C. John Langley, Jr. (1996), The Management of Business Logistics, Sixth Edition, West: Minneapolis/St. Paul.

Dunlop, John T., and Jan W. Rivkin (1997), "Introduction," in Revolution at the Checkout Counter, Harvard University Wertheim Committee: Cambridge, Mass.

Fang, Bingsong; Xiaoli Han; Ann M. Lawson; and Sherlene K.S. Lum (1998), "U.S. Transportation Satellite Accounts for 1992," Survey of Current Business 78(4), 16-27.

Hwang, Margaret Y., and David Weil (1998), "The Diffusion of Modern Manufacturing Practices: Evidence From the Retail-Apparel Sectors," Manuscript, Boston University.

Kahn, Barbara E., and Leigh McAlister (1997), Grocery Revolution: The New Focus on the Consumer, Addison-Wesley: Reading, Mass.

Kinsey, Jean; Ben Senauer; Robert P. King; and Paul F. Phumpiu (1996), "Changes in Retail Food Delivery: Signals for Producers, Processors and Distributors," Retail Food Industry Center Working Paper 96-03, University of Minnesota.

Kumar, Nirmalya (1996), "The Power of Trust in Manufacturer-Retailer Relationships," Har- 
vard Business Review 74(6), 92-106.

Milgrom, Paul, and John Roberts (1988), "Communication and Inventory as Substitutes in Organizing Production," Scandinavian Journal of Economics 90(3), 275-89.

Milgrom, Paul, and John Roberts (1990), "The Economics of Modern Manufacturing: Technology, Strategy, and Organization," American Economic Review 80 (3), 511-28.

Song, Jing-Sheng (1994), "The Effect of Leadtime Uncertainty in a Simple Stochastic Inventory Model," Management Science 40 (5), 603-13.

U.S. Bureau of the Census (1981), County Business Patterns 1978: United States, U.S. Government Printing Office: Washington, D.C.

U.S. Bureau of the Census (1997), County Business Patterns 1995: United States, U.S. Government Printing Office: Washington, D.C.

Vance, Sandra A., and Roy V. Scott (1994), Wal-Mart: A History of Sam Walton's Retail Phenomenon, Twayne: New York. 